\title{
Erratum to: Effect of Probiotic Bacillus Coagulans and Lactobacillus Plantarum on Alleviation of Mercury Toxicity in Rat
}

\author{
Majid Majlesi $^{1}$ • Seyed Shahram Shekarforoush ${ }^{1} \cdot$ Hamid Reza Ghaisari $^{1}$ • \\ Saeid Nazifi ${ }^{2}$. Javad Sajedianfard ${ }^{3}$ • Mohammad Hadi Eskandari ${ }^{4}$
}

Published online: 15 February 2017

(C) Springer Science+Business Media New York 2017

Erratum to: Probiotics \& Antimicro. Prot. DOI:10.1007/s12602-016-9250-x

The original version of this article contained mistakes and the authors are hereby publishing this erratum.

The author realized that the icon pattern of legend of Figs. 2 and 3 does not match to figures pattern. The correct legends are as follows:

Fig. 2 Effects of probiotics on the levels of mercury in feces and tissues of rat at day 24 (a) and 48 (b). Control

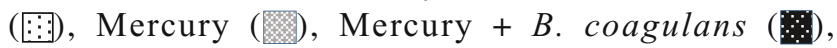
Mercury + L. plantarum ( $\mathbf{0})$. All results are expressed as mean $\pm \mathrm{SD}$ of three rats in each group. The different letters indicate statistically significant differences between groups $(P<0.05)$

Fig. 3 Effects of mercury and probiotics on bacterial population of stool, (a total count, b Anaerobe count, $\mathbf{c}$ LAB count, $\mathbf{d}$ L. plantarum count, e B. coagulans count). Control (::), L. plantarum treatment (W), B. coagulans treatment (B), Mercury treatment $(\mathbf{Q})$, Mercury $+B$. coagulans treatment $($ ), Mercury + L. plantarum treatment (ख). All results are expressed as mean $\pm \mathrm{SD}$ of three rats in each group. The different letters indicate statistically significant differences between groups in each day $(P<0.05)$

The online version of the original article can be found at http://dx.doi.org/ 10.1007/s12602-016-9250-x

Seyed Shahram Shekarforoush

shekar@shirazu.ac.ir

Department of Food Hygiene and Public Health, School of Veterinary Medicine, Shiraz University, Shiraz, Iran

2 Department of Clinical Pathology, School of Veterinary Medicine, Shiraz University, Shiraz, Iran

3 Department of Physiology, School of Veterinary Medicine, Shiraz University, Shiraz, Iran

4 Department of Food Science and Technology, College of Agriculture, Shiraz University, Shiraz, Iran 